\title{
Étude de la stabilité oxydative de l'huile d'olive vierge extra tunisienne au cours de son stockage
}

\author{
Imène Ben TEKAYA ${ }^{1}$ \\ Mnasser HASSOUNA ${ }^{2}$ \\ ${ }^{1}$ Institut National Agronomique de Tunisie \\ (INAT), Laboratoire de technologie alimentaire, \\ 43, Avenue Charles Nicole 1082 Tunis \\ Mahrajène - Tunisie \\ <ibentekaya@yahoo.fr> \\ 2 École Supérieure des Industries Alimentaires \\ de Tunis (ESIAT), 58, Avenue Alain Savary \\ 1003 El Khadra Tunis - Tunisie
}

\begin{abstract}
Tunisian extra virgin olive oil (EVOO) of Chetoui variety oxidation intensity depends on intrinsic composition, model packing and storage conditions. Thus, external factors such as kind of packing (glass, terephtalate polyethylene (PET), metal), full or attenuated day light, obscurity, air on bottles headspace, presence of metallic trace (iron and copper) and filtration influence on EVOO oxidative stability during two years storage at ambient temperature was studied. Peroxide value and ultraviolet specific extinction at 232 and $270 \mathrm{~nm}\left(K_{232}\right.$ and $\left.K_{270}\right)$ of oil bottled in PET and stored at obscurity for two years are significantly then the highest. On the other hand, oil bottled in glass bottles has shown the best physicochemical and organoleptic characteristics and also phenol and $\alpha$-tocopherol contents were few higher than those of oil bottled into metal or PET packing. Oil exposure to light at storage increases quickly their photooxidation depending on EVOO chlorophylls levels. However, neither packing with nitrogen headspace nor filtration affects significantly oil stability.
\end{abstract}

Key words: extra virgin olive oil, packing, oxidative stability, storage, filtration, nitrogen, quality

\section{Introduction}

La production d'olive et sa transformation en huile ont un impact socio-économique très important en Tunisie, deuxième pays à la fois producteur mondial et exportateur d'huile d'olive vierge (HOV) après l'Union européenne. II est donc impératif pour la Tunisie, du point de vue économique, de garantir des conditions optimales pour obtenir des huiles de haute qualité. Cependant, à l'instar d'autres huiles végétales, les HOV subissent une oxydation pendant leur stockage, résultant de l'auto-oxydation et de la photo-oxydation. L'auto-oxydation dépend de plusieurs facteurs qui sont, entre autres, le degré d'insaturation de l'huile, les acides gras libres, la présence de traces métalliques et d'eau, l'emballage utilisé, la température ambiante, l'oxygène de l'atmosphère et l'exposition à la lumière du jour pour les emballages transparents. En revanche, la photooxydation est affectée par la quantité totale de pigments chlorophylliens et d'antioxydants naturels (bêtacarotène, tocophérols, phénols) contenus dans I'HOV [1-6]. Par ailleurs, la filtration, opération généralement effectuée une seule fois, et ce avant la mise à la consommation de l'huile afin d'assurer la limpidité du produit en éliminant les colloïdes hydratés qui y sont dispersés, peut également altérer la qualité de l'huile d'olive du fait qu'il engendre une exposition excessive de I'huile à l'air [7].

L'action directe de l'oxygène et l'action indirecte des autres facteurs qui permettent à l'oxygène de se fixer sur les acides gras entraînent l'oxydation de l'huile. Cette réaction se déroule en plusieurs étapes et comprend les réactions d'initiation, de propagation et de terminaison. Au cours de la phase d'initiation, l'absorption de l'oxygène est faible et ce ne sont donc que des réactions d'oxydation primaire qui interviennent en produisant des hydroperoxydes. Au cours de la phase de propagation, l'absorption de l'oxygène s'intensifie et les réactions d'oxydation primaire s'accélèrent à travers des mécanismes radicalaires en chaîne. Dès que cette phase de propagation a démarré, les réactions d'oxydation secondaire se déclenchent, les radicaux libres se scindent pour former des composés non radicalaires qui affectent la qualité de l'huile et représentent la cause principale de l'altération du goût et de l'odeur de l'huile [7, 2].

Ainsi, l'objectif du présent travail a été d'étudier l'influence de certaines conditions de stockage, telles que le type d'emballage utilisé (verre, métal, PET), l'exposition à la lumière totale du jour ou atténuée, l'obscurité, la filtration, le conditionnement sous azote et la présence de traces métalliques sur la stabilité oxydative des HOVE tunisiennes au cours de deux ans de conservation à température ambiante.

\section{Matériel et méthodes}

\section{Traitement des échantillons d'huile d'olive vierge}

L'étude a porté sur 100 L de trois HOVE (notées A, B et C) de la campagne oléicole 2000/2001 de la variété Chétoui, variété cultivée dans la région du nord de la Tunisie et représentant le principal cultivar de l'oliveraie tunisienne à côté de la variété Chemlali (centre et sud). Ces huiles ont été 
obtenues par un système continu à trois phases (centrifugation) et collectées dans une huilerie moderne, située au nord-est de la Tunisie et choisie sur la base de la qualité et de la régularité des huiles obtenues. Les huiles $A$ et $B$ ont été mises en bouteilles dès leur obtention dans trois types d'emballage : bouteilles en verre de $1 \mathrm{~L}$ de contenance, gallons métalliques de 3,785 L et bouteilles en PET de 0,5 L. Pour étudier l'effet du type d'emballage sur la stabilité oxydative des huiles, les échantillons ont été conservés à température ambiante et à l'obscurité pendant deux ans. Toutes les déterminations ont été effectuées pendant les périodes suivantes : (i) avril-juillet-octobre 2001, (ii) janvier-avril-juilletoctobre 2002 et (iii) janvier-avril 2003.

L'influence de la lumière sur la vitesse d'oxydation de l'huile a également été suivie périodiquement pendant cette même période de stockage. Ainsi, les huiles ( $A$ et $B$ ) ont été conservées dans des bouteilles en verre et maintenues à l'obscurité, à la lumière atténuée, les bouteilles ont ainsi été enveloppées par du papier aluminium en laissant une fente de $2 \mathrm{~cm}$ de hauteur sur tout leur pourtour, ou enfin exposées à la lumière totale du jour.

La filtration de l'huile, qui est de nos jours effectuée par certaines huileries afin d'empêcher la formation d'un dépôt au fond des bouteilles et de donner un certain éclat à l'huile destinée à l'exportation, peut éventuellement affecter ou retenir certains antioxydants. Son impact sur l'autooxydation et la photoxydation de l'huile a également été suivi. La filtration réalisée est une double filtration sur coton et sur papier filtre qui a été effectuée au laboratoire. Les échantillons d'huile conditionnés dans des bouteilles en verre de $0,5 \mathrm{~L}$ ont été entreposés à température ambiante et à l'obscurité, à lumière totale ou atténuée du jour (fente de $1 \mathrm{~cm}$ ). Toutefois, I'huile utilisée (B) a été préalablement exposée pendant une quinzaine de jours à la lumière.

Par ailleurs, l'effet de l'air contenu dans l'espace de tête des bouteilles en verre $(0,5 \mathrm{~L})$ sur la stabilité oxydative a également été évalué sur des échantillons d'huile (B), préalablement conditionnés sous azote et conservés à température ambiante et à l'obscurité.

Enfin, un suivi de l'oxydation accélérée a également été réalisé sur des échantillons d'huile (C) conditionnés dans des flacons en verre de $90 \mathrm{~mL}$, totalement remplis pour réduire l'espace de tête, et entreposés dans des étuves réglées à 40,50 et $60^{\circ} \mathrm{C}$ pendant respectivement, 27, 20 et 30 jours.

\section{Analyses physicochimiques et biochimiques des huiles d'olive vierges}

L'acidité libre [8], exprimée en pourcentage d'acide oléique, l'indice de peroxyde [9], I'indice de saponification [10], I'indice de réfraction [11] et I'indice d'iode [12] ont été mesurés. Les coefficients des extinctions spécifiques dans l'ultraviolet à $232 \mathrm{~nm}\left(\mathrm{~K}_{232}\right)$ et $270 \mathrm{~nm}\left(\mathrm{~K}_{270}\right)$ ont été déterminés selon la norme tunisienne [13] à l'aide d'un spectrophotomètre Shimadzu UV-1201. Le taux de chlorophylles totales [14] a été mesuré à l'aide d'un spectrophotomètre Shimadzu UV-1201 et les teneurs en hydroxytyrosol et en tyrosol ont été mesurées par chromatographie liquide-haute performance (CLHP) selon la méthode décrite par Cortesi et al. [15] à l'aide d'un chromatographe HP séries 1100 équipé d'un détecteur UV réglé à $280 \mathrm{~nm}$ et d'une colonne ODS C18 à phase inverse de $25 \mathrm{~cm}$ de longueur et de 4,6 $\mathrm{mm}$ de diamètre interne. Le débit est réglé à $1 \mathrm{~mL} / \mathrm{min}$ et la température à $21^{\circ} \mathrm{C}$. Les solvants d'élution sont composés d'un solvant $A$, mélange eau/acide acétique $(99,5 / 0,5$; $v / v)$, et d'un solvant $B$, l'acétonitrile $(A / B ; 90 / 10 ; v / v)$. L'étalon interne utilisé, l'acide syringique à raison de $1,9 \mathrm{mg} / 100 \mathrm{~mL}$, est préparé dans un mélange méthanol/eau $(80 / 20 ; \mathrm{v} / \mathrm{v})$. L'extraction des phénols a été effectuée à l'aide d'une solution méthanol/eau $(80 / 20 ; v / v)$. L' $\alpha$ tocophérol a été dosé par la méthode CLHP à détection fluorimétrique [16] à l'aide d'un chromatographe HP séries 1100 équipé d'un fluorimètre programmable HP $1046 \mathrm{~A}$ et d'une colonne de silice de $25 \mathrm{~cm}$ de longueur et de 4,6 mm de diamètre interne. La longueur d'onde d'excitation est de $290 \mathrm{~nm}$ et celle d'émission est de $330 \mathrm{~nm}$. Le débit est réglé à $1 \mathrm{~mL} / \mathrm{min}$ et la température à $21^{\circ} \mathrm{C}$. Le solvant d'élution est un mélange hexane-propan-2-ol $(99,5 / 0,5 ; \mathrm{v} / \mathrm{v})$. L'étalon externe utilisé est le DL- $\alpha$-tocophérol (Merck). Le temps d'induction de I'oxydation primaire (en h) a également été mesuré [17] par Rancimat (Rancimat Metrohm 743). Les conditions opératoires ont été les suivantes: une prise d'essai d'huile $C$ de $3 \mathrm{~g}$, un débit d'air de $20 \mathrm{~L} / \mathrm{h}$ et une température de réaction de $120^{\circ} \mathrm{C}$.

La détermination de la composition en acides gras totaux des huiles (A et B) a été réalisée en préparant les esters méthyliques selon la norme internationale ISO 5509 [18]. Ces esters ont ensuite été analysés par chromatographie en phase gazeuse (CPG) [19] à l'aide d'un chromatographe Shimadzu GC14B à détecteur à ionisation de flamme (FID), équipé d'une colonne capillaire Carbowax (phase stationnaire polyéthylène glycol) de $20 \mathrm{~m}$ de longueur et de $0,25 \mathrm{~mm}$ de diamètre intérieur et d'un intégrateur-enregistreur CR7A. La température du four est réglée à $190^{\circ} \mathrm{C}$, celle de l'injecteur à $230^{\circ} \mathrm{C}$ et celle du détecteur est à $230^{\circ} \mathrm{C}$. Le gaz vecteur utilisé est l'hélium de $0,5 \mathrm{~mL} / \mathrm{min}$ et le volume d'injection est de $0,3 \mu \mathrm{L}$.

La composition en triglycérides a été déterminée par CLHP à détection par indice de réfraction [20] sur un chromatographe liquide hauteperformance de type HP séries 1100 équipé d'une colonne ODS C18 à phase inverse de $25 \mathrm{~cm}$ de longueur et de $4,6 \mathrm{~mm}$ de diamètre interne ; le détecteur est un réfractomètre de type HP 1047A. Le solvant d'élution utilisé est l'acétonitrile/acétone $(50 / 50 ; \mathrm{v} / \mathrm{v})$. La composition stérolique a été déterminée selon la norme $\mathrm{COI}$ [21] par un chromatographe en phase gazeuse HP6890 (GC system) à détecteur FID, équipé d'une colonne capillaire (phase stationnaire $5 \%$ phényl méthyl silicone) de $30 \mathrm{~m}$ de longueur, de $0,32 \mathrm{~mm}$ de diamètre intérieur et de $0,25 \mu \mathrm{m}$ d'épaisseur de film. La température du four est réglée à $260^{\circ} \mathrm{C}$, celle de I'injecteur à $280^{\circ} \mathrm{C}$ et celle du détecteur à $290^{\circ} \mathrm{C}$. Le gaz vecteur utilisé est l'hélium avec un débit de $0,9 \mathrm{~mL} / \mathrm{min}$ et le volume d'injection est de $10 \mu \mathrm{L}$. La composition en alcools aliphatiques [22] a été déterminée selon le même procédé et à l'aide du même appareillage utilisé pour la détermination de la composition stérolique, seules les conditions opératoires sont différentes. Ainsi, la température du four est alors réglée à $180^{\circ} \mathrm{C}$ pendant $8 \mathrm{~min}$ puis augmentée de $5^{\circ} \mathrm{C} / \mathrm{min}$ jusqu'à atteindre $280^{\circ} \mathrm{C}$ puis maintenue à $280^{\circ} \mathrm{C}$ pendant $30 \mathrm{~min}$; celle de l'injecteur est de $200^{\circ} \mathrm{C}$ et celle du détecteur est de $300^{\circ} \mathrm{C}$. Le gaz vecteur utilisé est I'hélium avec un débit de $1,5 \mathrm{~mL} / \mathrm{min}$ et le volume d'injection est de 10 $\mu \mathrm{L}$.

Après les deux années de conservation des huiles, le dosage des traces métalliques (fer et cuivre) a également été effectué par spectrométrie $d^{\prime}$ 'absorption atomique avec four en graphite [23] de type AAS VARIO 6. Les longueurs d'onde ont été ajustées à 302,1 et 324,7 nm, respectivement pour le dosage du fer et du cuivre.

Chaque prélèvement dans le temps a été effectué sur une nouvelle bouteille (modèle « distributeur ») et toutes les déterminations ont été effectuées en double pendant toute la durée de stockage des huiles étudiées.

\section{Analyses sensorielles des huiles d'olive vierges}

Après les deux années de conservation, l'évaluation organoleptique des huiles a été réalisée selon la norme COI [24] par 8 dégustateurs qualifiés de l'office national de l'huile de Tunisie. Les conditions des essais ont été choisies selon la norme COI [25], à savoir l'échantillon d'huile à analyser est présenté dans les verres de dégustation à une température de $28^{\circ} \mathrm{C} \pm 2{ }^{\circ} \mathrm{C}$. Chaque dégustateur a ainsi flairé puis dégusté chaque huile et porté sur la feuille de profil mise à sa disposition l'intensité à laquelle il a perçu chacun des attributs négatifs (chômé, moisi-humide, lies, vineuxvinaigré, métallique, rance) et positifs (fruité, amer, piquant). Le respon- 
sable du jury, après avoir recueilli les feuilles de profil remplies par chacun des dégustateurs, a contrôlé les intensités attribuées et calculé les médianes des différents attributs [24].

\section{Analyses statistiques}

La régression linéaire simple du type $(y=a+b x)$ a été utilisée pour déterminer la relation entre la variable à expliquer (y) (acidité libre de la matière grasse en $\%$ d'acide oléique) et la variable explicative $(x)$ (durée de stockage en mois) à l'aide d'un logiciel statistique SPSS version 8.0. Les résultats relatifs à l'évolution des critères de qualité des HOVE $A$ et $B$ (acidité libre, indice de peroxyde, $\mathrm{K}_{232}, \mathrm{~K}_{270}$ et teneur en chlorophylles totales) en fonction des conditions de stockage (type d'emballage utilisé, lumière totale ou atténuée du jour, obscurité, air contenu dans l'espace de tête des bouteilles et filtration) ont été comparés deux à deux par le test $t$ de Student [26], et ceci durant deux ans de stockage.

Les différences au seuil $p<0,05$ ont été considérées comme statistiquement significatives.

\section{Résultats et discussion}

\section{Caractéristiques initiales des huiles d'olive vierges}

L'examen de la composition initiale des huiles étudiées (tableaux $1 \mathrm{~A}$ et 1B) a permis de les classer toutes trois en huiles d'olive vierges extra [27]. $L$ 'huile $B$ a présenté toutefois les meilleurs critères de qualité. Ainsi, cette huile a montré l'acidité libre, $K_{232}$ et $K_{270}$ et l'indice de peroxyde les plus faibles. De plus, ses taux de stérols et de chlorophylles totales sont plus élevés par rapport à I'huile $A$. Cependant, ses teneurs en $\beta$-carotène, phénols et en $\alpha$-tocophérol sont les plus faibles. Enfin, toutes les HOVE tunisiennes étudiées se sont caractérisées par leur relative richesse en acides linoléique et palmitique et par leur faible teneur en acide oléique, contrairement aux huiles espagnoles, italiennes et grecques [5].

\section{Influence de certaines conditions de stockage sur la stabilité oxydative des huiles d'olive vierges}

\section{Emballage}

L'acidité libre, facteur qui renseigne sur l'altération de l'huile par hydrolyse, évolue linéairement de manière significative $(p<0,01)$ (résultats non montrés) en fonction de la durée de stockage des huiles (figure 1). Les relations linéaires trouvées entre l'acidité libre A (\%) de I'huile A conditionnée dans les trois types d'emballage et la durée de stockage $(\mathrm{t}$ en mois) sont : $\mathrm{A}(\%)=0,0174 t+0,49$ (verre), $\mathrm{A}(\%)=0,0179 t+0,49$ (plastique) et $\mathrm{A}(\%)=0,0189 \mathrm{t}+0,49$ (métal). En ce qui concerne l'huile $B$, les droites deviennent respectivement: $A(\%)=0,0181 \mathrm{t}+0,37$, A $(\%)=0,017 t+0,37$ et $A(\%)=0,0184 t+0,37$. Toutefois, aucune différence significative de l'acidité n'apparait entre les huiles conditionnées dans les trois types d'emballages. Par ailleurs, l'étude de l'évolution de I'acidité libre de l'huile $C$ en fonction de la durée d'étuvage ( $t$ ' en jours) à différentes températures (figure 2 ) a également montré une augmentation à tendance linéaire de ce paramètre en fonction du stade de conservation pour une température donnée. Ainsi, à 40 et $50^{\circ} \mathrm{C}$, I'acidité évolue de la même façon, l'équation de la droite étant $A$ $(\%)=0,0013 t^{\prime}+0,66$. Lorsque la température d'étuvage est fixée à $60^{\circ} \mathrm{C}$, l'acidité augmente plus rapidement en suivant l'équation: A $(\%)=0,0097 t^{\prime}+0,73$.

L'examen des résultats relatifs à l'évolution de l'indice de peroxyde (figure 3) et du $\mathrm{K}_{232}$ (figure 4 ) au cours du stockage a permis de montrer que ces paramètres deviennent plus élevés dans les huiles conditionnées dans les bouteilles en PET à partir de 12 mois de stockage pour l'huile $A$ $(p<0,05)$ et 18 mois pour l'huile $B(p<0,01)$, ce qui suggère que l'emballage en plastique favorise l'oxydation primaire, en raison proba-
Tableau 1A. Composition initiale des huiles d'olives vierges extra tunisiennes (huiles $A$, $B$ et $C$ ).

\begin{tabular}{|c|c|c|c|}
\hline Caractéristiques & Huile A & Huile B & Limites [28] \\
\hline $\begin{array}{l}\text { Acidité } \\
\text { (\% acide oléique) }\end{array}$ & $0,49 \pm 0,01$ & $0,37 \pm 0,01$ & $\leq 1,0$ \\
\hline$K_{232}$ & $2,50 \pm 0,01$ & $2,08 \pm 0,04$ & \\
\hline$K_{270}$ & $0,21 \pm 0,01$ & $0,17 \pm 0,01$ & $\leq 0,25$ \\
\hline$\Delta K$ & $<0$ & $<0$ & $\leq 0,01$ \\
\hline $\begin{array}{l}\text { Indice de peroxyde (méq } \\
\mathrm{O}_{2} \text { actif/kg) }\end{array}$ & $11,4 \pm 0,6$ & $8,1 \pm 0,4$ & $\leq 20$ \\
\hline $\begin{array}{l}\text { Indice d'iode } \\
(\mathrm{g} / 100 \mathrm{~g})\end{array}$ & $90,94 \pm 0,18$ & $90,09 \pm 0,17$ & \\
\hline $\begin{array}{l}\text { Indice de saponification } \\
\text { (mg de } \mathrm{KOH} / \mathrm{g})\end{array}$ & $203,3 \pm 1,1$ & $194,9 \pm 1,4$ & \\
\hline $\begin{array}{l}\text { Indice de réfraction } \\
\left(\grave{a} 20^{\circ} \mathrm{C}\right)\end{array}$ & 1,469 & 1,470 & \\
\hline$\beta$-carotène (ppm) & 2,7 & 1,5 & \\
\hline $\begin{array}{l}\text { Chlorophylles totales } \\
\text { (ppm) } \\
\text { Temps d'induction de } \\
\text { I'oxydation primaire (h) à } \\
120^{\circ} \mathrm{C}\end{array}$ & $2,46 \pm 0,01$ & $4,46 \pm 0,01$ & \\
\hline \multicolumn{4}{|l|}{ Acides gras (\%) } \\
\hline$C_{16}: 0(P)$ & $10,76 \pm 0,01$ & $11,18 \pm 0,33$ & $7,5-20,0$ \\
\hline$C_{16}: 1$ & $0,45 \pm 0,01$ & $0,62 \pm 0,02$ & $0,3-3,5$ \\
\hline$C_{17}: 0$ & $0,03 \pm 0,01$ & $0,06 \pm 0,03$ & $\leq 0,3$ \\
\hline$C_{17}: 1$ & $0,05 \pm 0,00$ & $0,06 \pm 0,01$ & $\leq 0,3$ \\
\hline$C_{18}: 0(S)$ & $2,85 \pm 0,04$ & $2,60 \pm 0,06$ & $0,5-5,0$ \\
\hline$C_{18}: 1(0)$ & $67,39 \pm 0,30$ & $68,26 \pm 0,09$ & $55,0-83,0$ \\
\hline$C_{18}: 2(L)$ & $17,22 \pm 0,23$ & $15,89 \pm 0,18$ & $3,5-21,0$ \\
\hline$C_{18}: 3(\operatorname{Ln})$ & $0,60 \pm 0,06$ & $0,61 \pm 0,01$ & $\leq 1,0$ \\
\hline$C_{20}: 0(A)$ & $0,39 \pm 0,01$ & $0,38 \pm 0,05$ & $\leq 0,6$ \\
\hline$C_{20}: 1$ & $0,30 \pm 0,02$ & $0,36 \pm 0,01$ & $\leq 0,4$ \\
\hline \multicolumn{4}{|l|}{ Triglycérides (\%) } \\
\hline LLL & $0,64 \pm 0,04$ & $0,61 \pm 0,00$ & \\
\hline LlnO & $0,43 \pm 0,04$ & $0,41 \pm 0,01$ & \\
\hline $\operatorname{LnLP}$ & $0,12 \pm 0,02$ & $0,10 \pm 0,02$ & \\
\hline LLO & $6,48 \pm 0,48$ & $6,15 \pm 0,10$ & \\
\hline LnOO & $2,68 \pm 0,08$ & $2,65 \pm 0,14$ & \\
\hline PLL & $0,39 \pm 0,05$ & $0,38 \pm 0,04$ & \\
\hline LOO & $20,45 \pm 0.54$ & $19,81 \pm 0,36$ & \\
\hline LOP & $9,21 \pm 0,07$ & $9,55 \pm 0,39$ & \\
\hline PLP & $0,87 \pm 0,06$ & $1,10 \pm 0,17$ & \\
\hline 000 & $32,22 \pm 0,69$ & $32,14 \pm 0,99$ & \\
\hline POO & $18,00 \pm 0,43$ & $19,17 \pm 1,25$ & \\
\hline POP & $2,33 \pm 0,11$ & $2,37 \pm 0,05$ & \\
\hline ALO & $0,57 \pm 0,02$ & $0,42 \pm 0,15$ & \\
\hline SOO & $4,62 \pm 0,12$ & $4,25 \pm 0,27$ & \\
\hline SOP & $1,01 \pm 0,03$ & $0,91 \pm 0,10$ & \\
\hline
\end{tabular}

blement de sa perméabilité à l'oxygène de l'air. Par ailleurs, nous avons observé que l'indice de peroxyde et le $K_{232}$ des huiles ont présenté un premier pic après 4 à 5 mois et un deuxième pic après 12 à 15 mois de conservation à température ambiante. Des constatations similaires ont également été retrouvées lorsque l'huile $C$ est étuvée à hautes températures (figure 5). Ainsi, un premier pic a, dans ce cas, a été enregistré après 40 jours de stockage $\left(\mathrm{à}^{\circ} 0^{\circ} \mathrm{C}\right)$ puis un deuxième après 70 jours $\left(\right.$ à $60^{\circ} \mathrm{C}$ ). Ce résultat suggère, $d^{\prime}$ une part, que l'élévation de la température de stockage d'environ $10{ }^{\circ} \mathrm{C}$ accélère la vitesse d'oxydation primaire et, 
Tableau 1B. Composition initiale des huiles d'olive vierges extra tunisiennes (huiles $A, B$ et $C$ ) (suite).

\begin{tabular}{|c|c|c|c|}
\hline Caractéristiques & Huile A & Huile B & Limites [28] \\
\hline \multicolumn{4}{|l|}{ Stérols (\%) } \\
\hline Cholestérol & 0,11 & 0,07 & $\leq 0,5$ \\
\hline Campestérol & 2,48 & 2,36 & $\leq 4,0$ \\
\hline Stigmastérol & 1,13 & 0,61 & $\leq$ Campestérol \\
\hline Clérostérol & 0,62 & 0,31 & \\
\hline$\beta$-sitostérol & 81,72 & 79,53 & \\
\hline $\delta$-avénastérol & 12,78 & 16,34 & \\
\hline \$5-24-stigmastadiénol & 0,57 & 0,25 & \\
\hline §7-stigmasténol & 0,23 & 0,22 & \\
\hline §7-avénastérol & 0,34 & 0,32 & \\
\hline \multicolumn{4}{|l|}{ Erythrodiol + uvaol } \\
\hline (\% par rapport aux stérols totaux) & 1,12 & 1,33 & $\leq 4,5$ \\
\hline Stérols totaux (ppm) & 1703 & 2285 & $\geq 1000$ \\
\hline \multicolumn{4}{|l|}{ Alcools aliphatiques (\%) } \\
\hline $\mathrm{C}_{22}$ Docosanol & 13,28 & 10,69 & \\
\hline $\mathrm{C}_{24}$ Tétracosanol & 23,39 & 23,59 & \\
\hline $\mathrm{C}_{26}$ Hexacosanol & 40,12 & 42,80 & \\
\hline $\mathrm{C}_{28}$ Octacosanol & 23,21 & 22,92 & \\
\hline Alcools aliphatiques totaux (ppm) & 113 & 164 & \\
\hline \multicolumn{4}{|l|}{ Composés phénoliques (ppm) } \\
\hline Hydroxytyrosol & $13,73 \pm 1,03$ & $3,16 \pm 0,13$ & \\
\hline Tyrosol & $15,43 \pm 0,04$ & $4,50 \pm 0,18$ & \\
\hline \multicolumn{4}{|l|}{ Tocophérols (ppm) } \\
\hline \multirow[t]{2}{*}{$\alpha$-tocophérol } & $448 \pm 20,5$ & $425 \pm 5,10$ & \\
\hline & \multicolumn{2}{|c|}{ Huile C } & \\
\hline Acidité(\%) & \multicolumn{2}{|c|}{$0,67 \pm 0,01$} & $\leq 1,0$ \\
\hline$K_{232}$ & \multicolumn{2}{|c|}{$2,17 \pm 0,07$} & \\
\hline$K_{270}$ & \multicolumn{2}{|c|}{$0,20 \pm 0,01$} & $\leq 0,25$ \\
\hline$\Delta K$ & \multicolumn{2}{|c|}{$<0$} & $\leq 0,01$ \\
\hline (méq $\mathrm{O}_{2}$ actif/kg) & \multicolumn{2}{|c|}{$12,8 \pm 0,5$} & $\leq 20$ \\
\hline Indice d'iode $(\mathrm{g} / 100 \mathrm{~g})$ & \multicolumn{2}{|c|}{$89,00 \pm 0,21$} & \\
\hline $\begin{array}{l}\text { Indice de saponification } \\
(\mathrm{mg} \text { de } \mathrm{KOH} / \mathrm{g})\end{array}$ & \multicolumn{2}{|c|}{$186,9 \pm 1,9$} & \\
\hline $\begin{array}{l}\text { Indice de réfraction }\left(\grave{a} 20^{\circ} \mathrm{C}\right) \\
\text { Tocophérols (ppm) }\end{array}$ & \multicolumn{2}{|c|}{1,470} & \\
\hline $\begin{array}{l}\alpha \text {-tocophérol (ppm) } \\
\text { Composés phénoliques (ppm) }\end{array}$ & \multicolumn{2}{|c|}{$515,07 \pm 18,30$} & \\
\hline Hydroxytyrosol (ppm) & \multicolumn{2}{|c|}{$44,13 \pm 1,00$} & \\
\hline Tyrosol (ppm) & \multicolumn{2}{|c|}{$19,89 \pm 0,23$} & \\
\hline
\end{tabular}

d'autre part, celle-ci se déroule par étapes. En effet, la vitesse relative d'oxydation des acides gras augmente avec le nombre d'insaturations. Ainsi, à $100^{\circ} \mathrm{C}$, les vitesses relatives d'oxydation des acides stéarique $\left(C_{18}: 0\right)$, oléique $\left(C_{18}: 1\right)$, linoléique $\left(C_{18}: 2\right)$ et linolénique $\left(C_{18}: 3\right)$ sont, par exemple, respectivement 1,100 et $1000-1500$ et $2000-3500$. L'acide linoléique, qui représente en moyenne 15 à $18 \%$ des acides gras totaux des huiles étudiées, s'oxyderait donc le premier puis l'acide oléique qui représente le composant majeur (67 à $69 \%$ ) et enfin l'acide stéarique $(2,5$ à $2,9 \%)$ [28, 2]. Par ailleurs, le $\mathrm{K}_{270}$ de l'huile A conditionnée dans le PET devient significativement supérieur à celui de ce même échantillon stocké dans les autres types d'emballage, et ceci à partir de 20 mois de conservation. Cette modification du $\mathrm{K}_{270}$ observée pour I'huile $A$ résulte de son oxydation primaire plus accentuée par rapport à celle notée pour I'huile $B$ (figure 6 ). Après deux ans de conservation à température ambiante, I'huile $A$, conditionnée dans les gallons métalli-

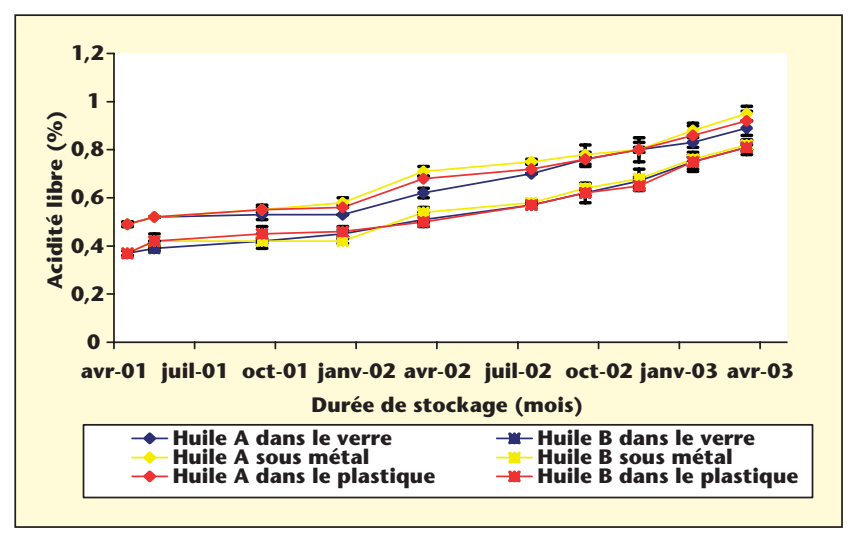

Figure 1. Évolution de l'acidité libre des huiles d'olive vierges extra tunisiennes conditionnées dans différents types d'emballages et stockées à température ambiante et à l'obscurité.

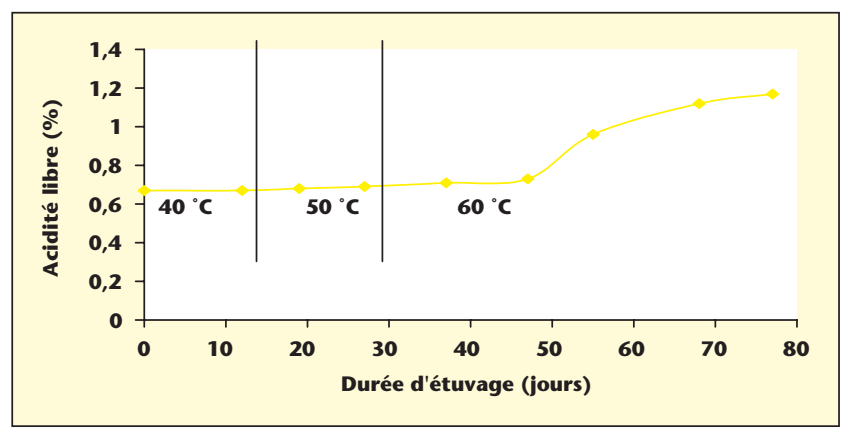

Figure 2. Évolution de l'acidité libre (\%) de l'huile d'olive vierge extra tunisienne (C) au cours de son étuvage à 40,50 et $60^{\circ} \mathrm{C}$ pendant respectivement, 27,20 et 30 jours.

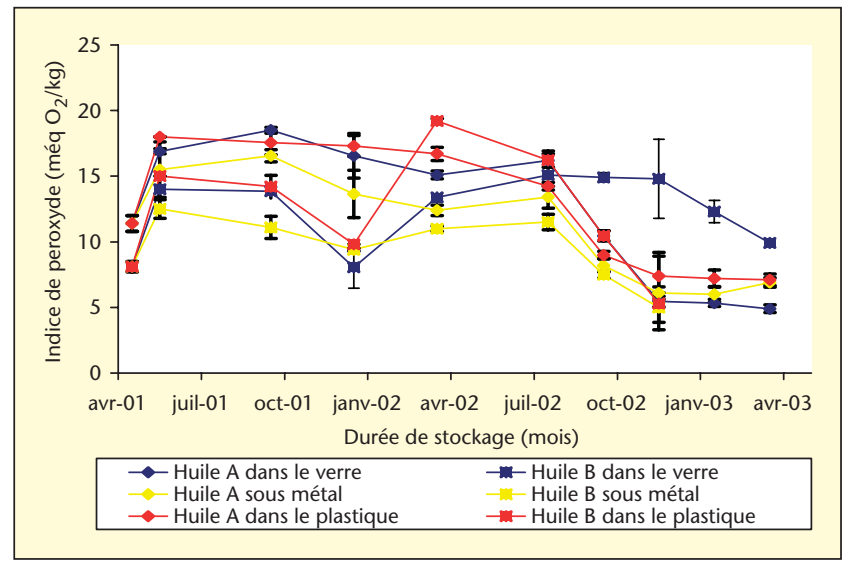

Figure 3. Évolution de l'indice de peroxyde (méq $\mathrm{d}^{\prime} \mathrm{O}_{2} / \mathrm{kg}$ ) des huiles d'olive vierges extra tunisiennes conditionnées dans différents types d'emballages et stockées à température ambiante et à l'obscurité.

ques, a présenté une flaveur de concombre, attribuée à la formation du 2-6 nonadiénal, qui se produit essentiellement suite à un conditionnement hermétique excessivement prolongé des huiles dans des récipients en fer-blanc [24], et ceci malgré la présence dans cet échantillon d'un taux de fer (20,10 ppb) inférieur au seuil fixé (3 ppm) [27]. Dans les huiles conditionnées dans le verre et dans le PET, ces teneurs deviennent respectivement égales à 15,42 et 14,35 ppb. Les quantité de cuivre n'ont 


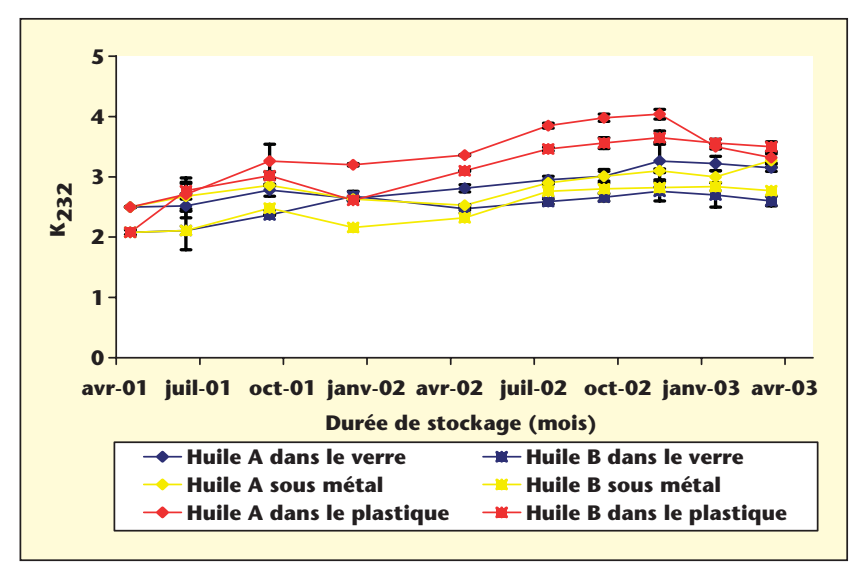

Figure 4. Évolution du $K_{232}$ des huiles d'olive vierges extra tunisiennes conditionnées dans différents types d'emballages et stockées à température ambiante et à l'obscurité.

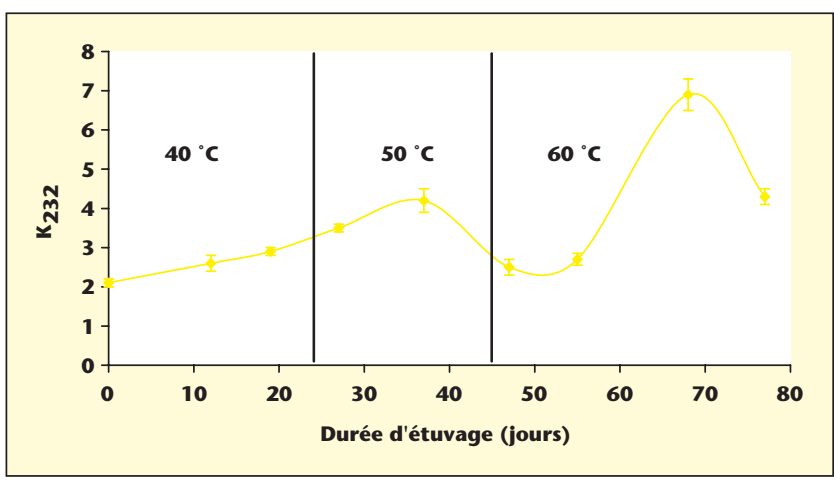

Figure 5. Évolution du $K_{232}$ de l'huile d'olive vierge extra tunisienne (C) au cours de son étuvage à 40,50 et $60^{\circ} \mathrm{C}$ pendant respectivement, 27,20 et 30 jours.

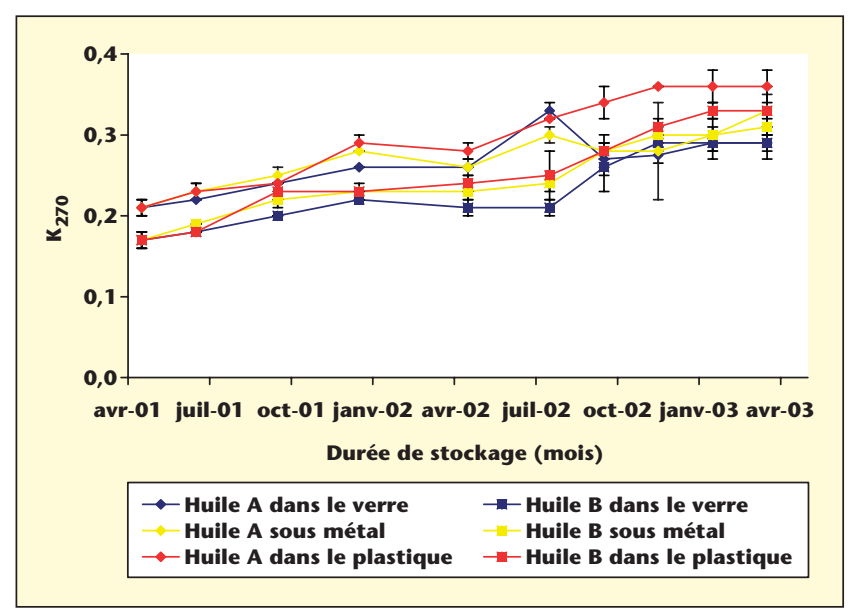

Figure 6. Évolution du $K_{270}$ des huiles d'olive vierges extra tunisiennes conditionnées dans différents types d'emballages et stockées à température ambiante et à l'obscurité.

pas été significativement modifiées et les valeurs mesurées sont de 11,30, 11,70 et 11,24 ppb, lorsque l'huile A a été conditionnée respectivement dans le verre, le métal et le PET. Ces concentrations restent également bien en dessous du seuil fixé $(0,1 \mathrm{ppm})$ par la norme commerciale COI T.15/NC Rév.10 [27]. Par ailleurs, le $\mathrm{K}_{270}$ augmente de manière régulière lorsque l'huile $\mathrm{C}$ a été étuvée à températures élevées (figure 7), comme cela a été observé pour les huiles $A$ et $B$ conservées à température ambiante et à l'obscurité. Cependant, nous avons constaté que tout au long de la phase de conservation, les valeurs trouvées pour l'huile $A$ restent toujours supérieures à celles mesurées pour I'huile B qui a présenté initialement de meilleures caractéristiques physicochimiques et biochimiques. L'huile $A$ a perdu sa dénomination d'HOVE après 9 mois de conservation, son $K_{270}$ dépassant les normes [27], alors que I'huile $B$ reste HOVE pendant 18 mois, résultat en accord avec le temps d'induction de l'oxydation primaire à $120^{\circ} \mathrm{C}$ de l'huile $\mathrm{A}(8 \mathrm{~h})$ qui était inférieur à celui de l'huile $B(9,3 \mathrm{~h})$. Après deux ans de stockage, l'évaluation organoleptique (tableau 2) de l'huile A conditionnée dans les trois types d'emballages a donné une médiane des défauts supérieure à 0 et inférieure à 2,5 et une médiane du fruité supérieure à 0 , ce qui permet de la classer en HOV [24]. Le défaut principal perçu dans les différents échantillons est la flaveur de «lies » caractéristique de l'huile restée en contact avec les « boues » de décantation dans les cuves. En outre, l'huile conditionnée dans les gallons métalliques a présenté une flaveur de moisi-humide.

Par ailleurs, les teneurs en $\alpha$-tocophérol (figure 8) et en composés phénoliques majoritaires (hydroxytyrosol et tyrosol) (figures 9 et 10) dans les huiles conditionnées dans les emballages en verre restent presque toujours supérieures à celles des autres échantillons conservés dans le plastique et dans le métal, ce qui confirme encore la meilleure stabilité oxydative des huiles conditionnées dans ce type d'emballage. Les teneurs en hydroxytyrosol et en tyrosol, anti-oxydants naturels, n'ont cessé d'augmenter pendant la première année de stockage de façon linéaire avec une pente plus accentuée pour l'hydroxytyrosol. Des résultats similaires ont été trouvés par Pagliarini et al. [29] sur des HOVE italiennes stockées pendant 21 mois environ à l'obscurité et à $20^{\circ} \mathrm{C}$. Cette augmentation s'expliquerait par l'hydrolyse de certaines substances phénoliques complexes, telles que l'oleuropéine qui libère l'hydroxytyrosol et la verbascoside qui libère l'acide caféique et I'hydroxytyrosol... [3]. En revanche, la teneur en $\alpha$-tocophérol a diminué pendant les trois premiers mois de stockage des huiles puis elle subit une augmentation durant une période de 9 à 10 mois pour diminuer à nouveau par la suite. On peut corréler la baisse du taux de la vitamine E à l'augmentation de l'indice de peroxyde des huiles. En effet, l'a-tocophérol possède une action antioxydante par rupture de la chaîne radicalaire lors des étapes de propagation de l'oxydation. II agit avec les radicaux hydroperoxyle par transfert d'hydrogène avec formation d'hydroperoxydes et de radicaux aryloxyle qui se couplent avec d'autres radicaux hydroperoxyle, dans une réaction de terminaison, pour former des produits non radicalaires. Par ailleurs, I'augmentation de la teneur en $\alpha$-tocophérol s'expliquerait par la régénération des vecteurs de propagation de chaîne en arrachant un hydrogène $d^{\prime}$ hydroperoxyde ou bis-allylique [30].

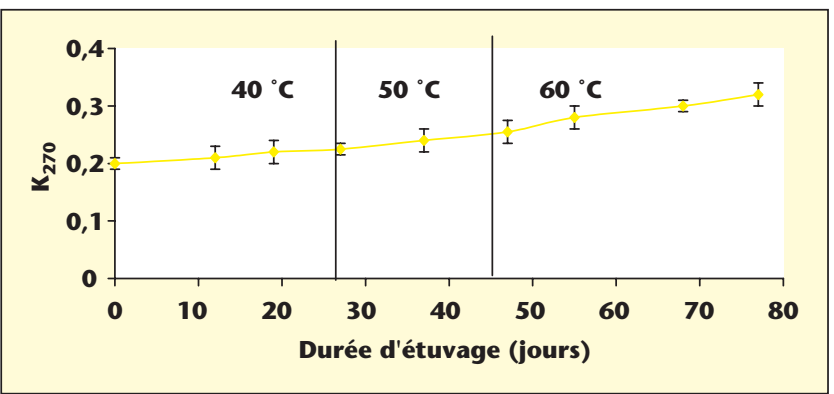

Figure 7. Évolution du $K_{270}$ de I'huile d'olive vierge extra tunisienne (C) au cours de son étuvage à 40,50 et $60^{\circ} \mathrm{C}$ pendant respectivement, 27,20 et 30 jours. 
Tableau 2. Évaluation organoleptique (médiane des attributs positifs et négatifs) des huiles d'olive vierges tunisiennes (A et B) après deux ans de conservation à température ambiante.

\begin{tabular}{|lccccccc|}
\hline & \multicolumn{3}{c}{ Attributs négatifs } & \multicolumn{3}{c|}{ Attributs positifs } \\
\cline { 2 - 8 } Facteur étudié & Type d'huile & Lies & Chômé & Moisi- humide & Fruité & Amer & Piquant \\
\hline Emballage & AVO & 2,2 & - & - & 2,0 & 3,0 & 2,2 \\
& AMO & 2,0 & - & 1,8 & 2,5 & 2,0 & 2,1 \\
\multirow{3}{*}{ Filtrage } & APO & 2,4 & - & - & 2,2 & 2,9 & 2,4 \\
& BNFVO & 3,0 & 4,0 & - & 1,4 & 2,0 & 2,1 \\
Lumière & BFVO & 3,2 & 4,2 & - & 1,4 & 2,5 & 2,0 \\
& BNFVO & 3,0 & 4,0 & - & 1,4 & 2,0 & 2,1 \\
& BNFVL & 3,2 & 4,1 & - & 1,5 & 2,0 & 2,0 \\
\hline
\end{tabular}

Abréviations : AVO : huile A conditionnée dans le verre et conservée à l'obscurité; AMO : huile A conditionnée dans le métal et conservée à l'obscurité; APO : huile A conditionnée dans le plastique et conservée à l'obscurité; BNFVO : huile B non filtrée conditionnée dans le verre et conservée à l'obscurité; BFVO : huile B filtrée conditionnée dans le verre et conservée à l'obscurité; BNFVL : huile B non filtrée conditionnée dans le verre et conservée à la lumière du jour.

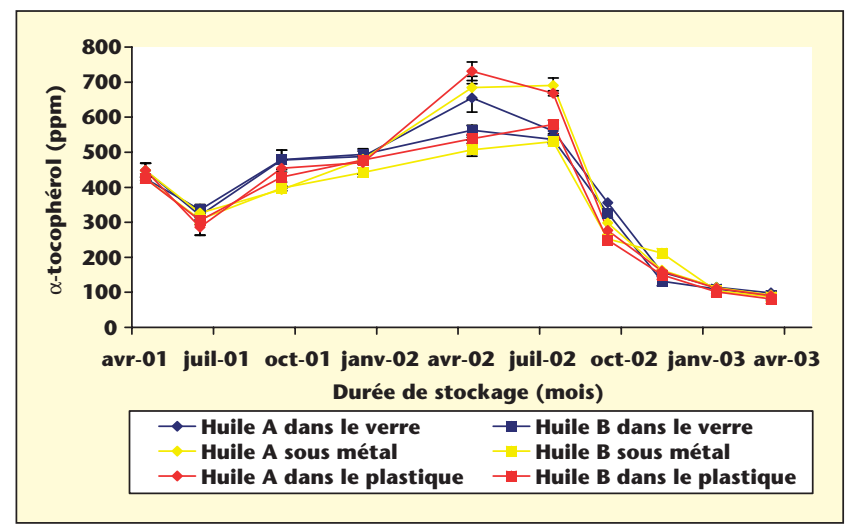

Figure 8. Évolution de la teneur en $\alpha$-tocophérol (ppm) des huiles d'olive vierges extra tunisiennes conditionnées dans différents types d'emballages et stockées à température ambiante et à l'obscurité.

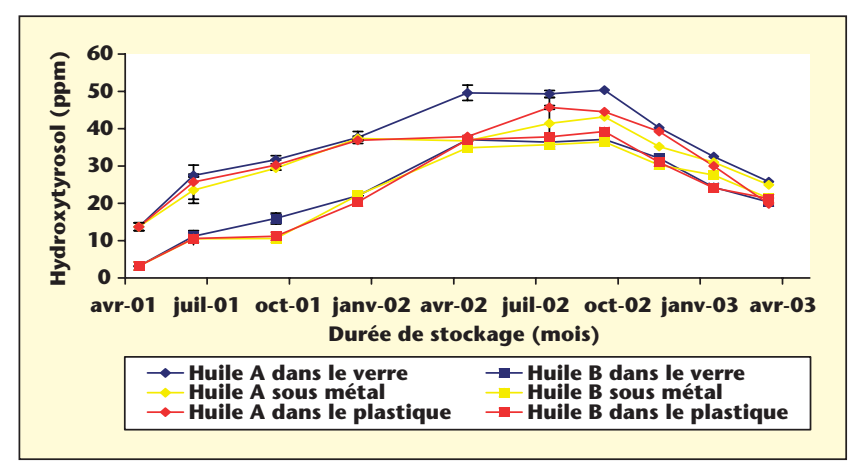

Figure 9. Évolution de la teneur en hydroxytyrosol (ppm) des huiles d'olive vierges extra tunisiennes conditionnées dans différents types d'emballages et stockées à température ambiante et à l'obscurité.

\section{Filtration et conditionnement sous azote}

La filtration et le conditionnement sous azote de l'huile $B$ (en bouteilles de verre) n'ont aucun effet significatif sur l'évolution du $K_{232}$ et du $K_{270}$ au cours de son stockage à température ambiante et à l'obscurité (figures 11 et 12). Le conditionnement sous azote ne prévient pas contre l'oxydation même au cours des premiers mois de conservation. L'oxygène contenu dans l'espace de tête n'est donc pas le facteur déterminant de l'initiation de l'oxydation, l'initiation étant déclenchée avant la mise en bouteilles. Toutefois, la durabilité de I'HOVE filtrée conditionnée sous azote est

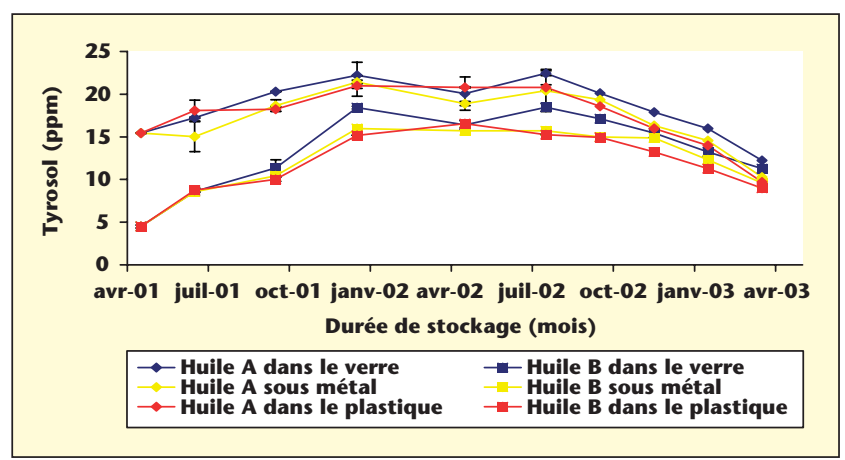

Figure 10. Évolution de la teneur en tyrosol (ppm) des huiles d'olive vierges extra tunisiennes conditionnées dans différents types d'emballages et stockées à température ambiante et à l'obscurité.

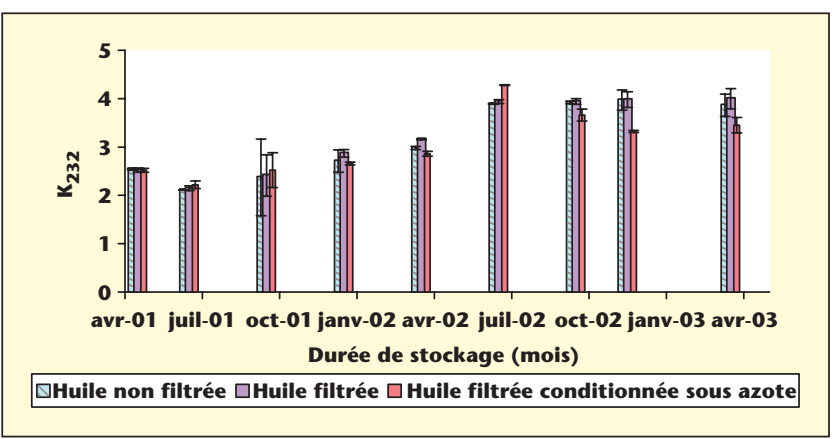

Figure 11. Évolution du $K_{232}$ des huiles d'olive vierges extra tunisiennes non filtrées, filtrées et filtrées conditionnées sous azote au cours de leur stockage à température ambiante et à l'obscurité.

supérieure d'environ un mois à celle de l'HOVE filtrée conditionnée sous air atmosphérique. L'évaluation organoleptique (tableau 2), effectuée au bout de deux ans de stockage des huiles filtrées et non filtrées, a permis de les classer en huiles d'olive vierges courantes puisque la médiane des défauts est supérieure à 2,5 et inférieure à 6,0 [24].

Enfin, nous avons noté que pendant toute la phase de conservation, la photo-oxydation reste un processus plus rapide dans les huiles filtrées que dans les huiles non filtrées (tableau 3), en raison notamment de la diminution importante de la teneur en stérols totaux (-582 ppm) suite à I'opération de filtration de I'huile B. Il a été rapporté que les stérols, et en particulier le $\beta$-sitostérol et le $\delta$ - 5 avénastérol, deux phytostérols majori- 
Tableau 3. Influence du filtrage sur l'évolution de la teneur en chlorophylles (ppm) au cours du stockage de l'huile d'olive vierge extra tunisienne (B) conditionnée dans des bouteilles en verre et conservée à l'obscurité, à la lumière atténuée et à la lumière totale du jour.

\begin{tabular}{|c|c|c|c|c|c|c|}
\hline \multirow{2}{*}{$\begin{array}{l}\text { Durée de stockage } \\
\text { (mois) }\end{array}$} & \multicolumn{3}{|c|}{ Huile non filtrée } & \multicolumn{3}{|c|}{ Huile filtrée } \\
\hline & Obscurité & Lumière atténuée & Lumière & Obscurité & Lumière atténuée & Lumière \\
\hline avr-01 & $4,47 \pm 0,00$ & $4,47 \pm 0,00$ & $4,47 \pm 0,00$ & $4,47 \pm 0,01$ & $4,47 \pm 0,01$ & $4,47 \pm 0,01$ \\
\hline juil-01 & $4,42 \pm 0,02$ & $3,02 \pm 0,03$ & $1,02 \pm 0,01$ & $4,40 \pm 0,03$ & $2,94 \pm 0,02$ & $0,89 \pm 0,02$ \\
\hline oct-01 & $4,00 \pm 0,03$ & $2,80 \pm 0,05$ & $0,45 \pm 0,04$ & $3,92 \pm 0,01$ & $2,46 \pm 0,01$ & $0,30 \pm 0,02$ \\
\hline janv-02 & $3,98 \pm 0,01$ & $2,68 \pm 0,02$ & $0,26 \pm 0,04$ & $3,88 \pm 0,02$ & $1,84 \pm 0,02$ & $0,18 \pm 0,03$ \\
\hline avr-02 & $3,97 \pm 0,03$ & $2,62 \pm 0,02$ & $0,30 \pm 0,05$ & $3,93 \pm 0,01$ & $1,37 \pm 0,02$ & $0,10 \pm 0,01$ \\
\hline juil-02 & $3,86 \pm 0,04$ & $2,60 \pm 0,03$ & $0,18 \pm 0,02$ & $3,80 \pm 0,03$ & $0,92 \pm 0,02$ & $0,07 \pm 0,01$ \\
\hline oct-02 & $3,84 \pm 0,02$ & $2,54 \pm 0,03$ & $0,12 \pm 0,02$ & $3,84 \pm 0,00$ & $0,74 \pm 0,03$ & $0,06 \pm 0,03$ \\
\hline janv-03 & $3,76 \pm 0,03$ & $2,32 \pm 0,02$ & $0,08 \pm 0,02$ & $3,69 \pm 0,00$ & $0,34 \pm 0,00$ & $0,05 \pm 0,03$ \\
\hline avr-03 & $3,68 \pm 0,02$ & $2,16 \pm 0,04$ & $0,10 \pm 0,03$ & $3,54 \pm 0,02$ & $0,08 \pm 0,01$ & $0,04 \pm 0,01$ \\
\hline
\end{tabular}

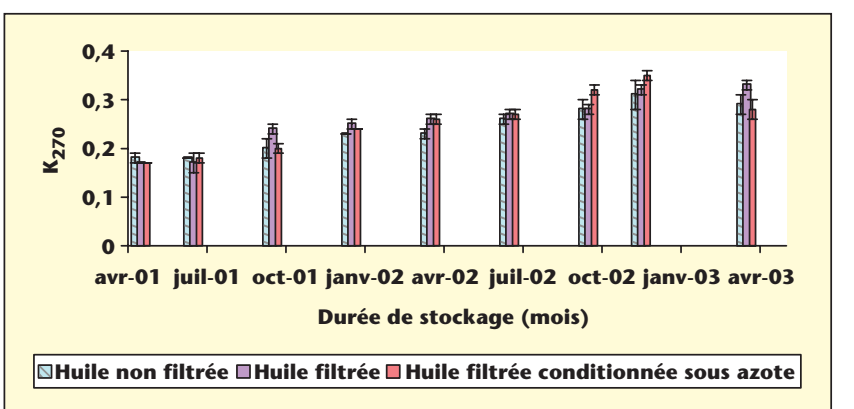

Figure 12. Évolution $d u K_{270}$ des huiles d'olive vierges extra tunisiennes non filtrées, filtrées et filtrées conditionnées sous azote au cours de leur stockage à température ambiante et à l'obscurité.

taires (96\% des stérols totaux) de l'HOVE B, sont doués de propriétés anti-oxydantes des huiles, notamment chauffées $[5,31]$

\section{Lumière}

Les échantillons d'huile A et B conservés à l'obscurité ont montré une meilleure stabilité oxydative tout au long de leur stockage en présentant, respectivement à partir de 18 et 20 mois de conservation un $\mathrm{K}_{232}$ et un $\mathrm{K}_{270}$ significativement plus faibles $(\mathrm{p}<0,05)$ par rapport à ceux exposés à la lumière du jour totale ou atténuée (figure 13). Toutefois, l'évaluation

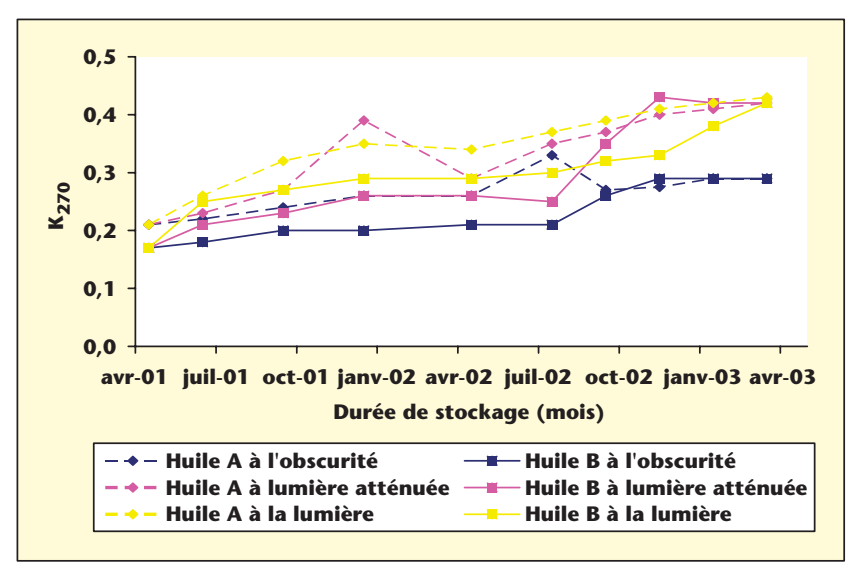

Figure 13. Évolution du $K_{270}$ des huiles d'olive vierges extra tunisiennes conservées à température ambiante, à l'obscurité, à la lumière atténuée et à la lumière du jour.

organoleptique (tableau 2), réalisée au bout de deux ans de stockage, a permis de classer aussi bien les huiles conservées à l'obscurité que celles stockées à la lumière sous la dénomination d'huiles d'olive vierges courantes. L'huile B, étant plus riche en chlorophylles que l'huile $A$, atteint, en fin de période de stockage à la lumière, le même état d'oxydation secondaire que I'huile A qui a présenté au départ un état d'oxydation plus avancé. Les pigments chlorophylliens dans les huiles végétales sont doués d'un pouvoir photosensibilisateur se traduisant par la production de l'oxygène singulet $\left({ }^{1} \mathrm{O}_{2}\right)$. Cette espèce $d^{\prime}$ 'oxygène excité, étant plus réactive envers les lipides insaturés que l'oxygène à l'état fondamental $\left({ }^{3} \mathrm{O}_{2}\right)$ dissout dans I'huile, aboutit à la formation d'hydroperoxydes. L'action pro-oxydante de ces pigments verts est, dans I'huile d'olive exposée à la lumière, fonction directe de leur concentration initiale et de leur type [1]. Par ailleurs, nous avons observé que les taux des pigments chlorophylliens dans les huiles (A et $B$ ) ont diminué rapidement pour atteindre, après trois mois de conservation à la lumière totale, des valeurs quasi-nulles, alors qu'à la lumière atténuée et à I'obscurité, cette chute est beaucoup moins rapide (figure 14). Toutefois,

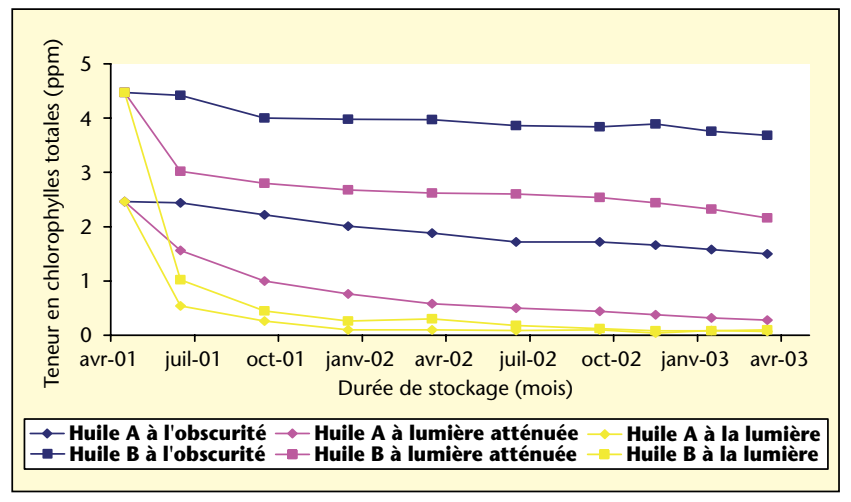

Figure 14. Évolution de la teneur en chlorophylles (ppm) des huiles d'olive vierges extra tunisiennes (A et B) conservées à température ambiante, à l'obscurité, à la lumière atténuée et à la lumière du jour.

pendant cette même phase de conservation, la dégradation des chlorophylles par blanchiment [32] reste un processus près de deux fois plus rapide dans I'huile $B$ que dans I'huile $A$, en rapport notamment avec sa teneur initiale plus élevée en pigments verts.

\section{Conclusion}

Les effets de certaines conditions de stockage sur l'évolution de la stabilité oxydative des huiles d'olive vierges extra tunisiennes ont été étudiés. 
Ainsi, la durée de vie des huiles étudiées varie de 9 à 18 mois en fonction de leurs caractéristiques initiales et des conditions d'entreposage appliquées. Le type d'emballage (verre, métal) n'a pas d'effet significatif sur la stabilité des HOVE (A et $B$ ) par comparaison au PET qui a occasionné une augmentation significative du $\mathrm{K}_{232}$ et du $\mathrm{K}_{270}$ en fin de stockage de I'huile $A$. Par ailleurs, la lumière accélère la photoxydation des huiles en réduisant significativement leurs teneurs en chlorophylles dès les trois premiers mois de stockage suite au phénomène de blanchiment et dont I'intensité est fonction du taux de ces pigments dans l'huile. En revanche, ni la filtration, ni le conditionnement sous azote ne permettent de retarder significativement le phénomène $d^{\prime}$ oxydation des huiles entreposées. Enfin, les effets de certains composés mineurs (chlorophylles, $\beta$-carotène, $\alpha$-tocophérol, tyrosol) sur la stabilité oxydative des huiles d'olive vierges et décolorées ont été étudiés à l'aide de plans factoriels complets et dont les résultats feront l'objet d'une publication ultérieure.

\section{RÉFÉRENCES}

1. RAHMANI M, SAAD L. Photooxydation des huiles d'olive : influence de la composition chimique. Rev Fr Corps Gras 1989 ; 36 : 355-60.

2. CHEFTEL JC, CHEFTEL H. Introduction à la biochimie et à la technologie des aliments. Paris : Lavoisier, 1992 ; (Tec et doc).

3. PERRIN JL. Les composés mineurs et les antioxygènes naturels de l'olive et de son huile. Rev Fr Corps Gras 1992 ; 39 : 25-32.

4. KIRITSAKIS A, OSMAN M. Effets du $\beta$-carotène et de I' $\alpha$-tocophérol sur la stabilité photooxydative de I'huile d'olive. Olivae $1995 ; 56: 25-8$.

5. BOSKOU D. Olive Oil Chemistry and Technology. Champaign, Illinois : AOCS Press, 1996.

6. RAHMANI M, SAARI CSALLANY A. Étude de la stabilité des huiles d'olive vierge marocaines. Olivae $2000 ; 82: 37-40$.

7. RANALLI A. Aspects et problèmes se rattachant au stockage et à la conservation de I'huile d'olive. Olivae $1989 ; 272$ : 11-8.

8. NT (Norme Tunisienne Enregistrée) 118. 18 (1994). Détermination de I'acidité de I'huile (graines oléagineuses) NT ISO 729 (1988).

9. NT 118.22 (1994). Détermination de l'indice de peroxyde (corps gras d'origines animale et végétale) NT ISO 3960 (1977).

10. NT 118.23 (1994). Corps gras d'origines animale et végétale. Détermination de l'indice de saponification. NT ISO 3657 (1988).

11. NT 118.76 (1994). Corps gras d'origines animale et végétale. Détermination de l'indice de réfraction. NT ISO 6320 (1985).

12. AOCS (American Oil Chemists Society) Officiel Method Da15-48 (1997) lodine Value, Wijs Method.

13. NT 118. 72 (1994). Détermination de l'absorbance dans l'ultraviolet (corps gras d'origines animale et végétale). NT ISO 3356 (1989).
14. AOCS. Official methods and recommended Practices. 4, ed. In : Champaign, Illinois : Firestone, 1987.

15. CORTESI N, ROVELLINI P, FEDELI E. Détermination des polyphénols par HPLC à détection UV. IOC Working Group 1. 1999.

16. POCKLINGTON WD, DIEFFENBACHER A. Determination of tocopherols and tocotrienols in vegetable oils and fats by high performance liquid chromatography: results of a collaborative study and the standardized method. Pure Appl Chem 1988 ; 60 : 877-92.

17. AOCS Official Method Cd 12b-92. Oil Stability Index (OSI). 1993.

18. ISO (Norme Internationale) 5509 Corps gras d'origines animale et végétale. Préparation de esters méthyliques d'acides gras. 1978.

19. ISO 5508. Analyse par chromatographie en phase gazeuse des esters méthyliques d'acides gras 1990.

20. JOCE (Journal Officiel des Communautés Européennes) nL248/29. Détermination de la teneur en trinoléine. 1991.

21. COI (Conseil Oléicole International) T. 20/Doc. $n^{\circ} 10 /$ Rév.1. Détermination de la composition et du contenu en stérols par chromatographie en phase gazeuse sur colonne capillaire. 2001.

22. JOCE $N^{\circ}$ L248/10. (Détermination du contenu en alcools aliphatiques au moyen de la chromatographie en phase gazeuse avec colonne capillaire. 1991.

23. AOCS Official Method Ca 18b-91 Determination of copper, iron and nickel by direct graphite furnace atomic absorption spectrometry. 1992.

24. COI T. 20/Doc. $n^{\circ} 15 /$ Rév.1. Évaluation organoleptique de l'huile d'olive vierge, 1-10. 1996.

25. COI T. 20/Doc. n¹3/Rév.1. Méthodologie générale pour l'évaluation organoleptique de I'huile d'olive vierge. 1996.

26. DAGNELIE P. Théorie et méthodes statistiques. Applications agronomiques, 2. ASBI, Gembloux : Les Presses Agronomiques de Gembloux, 1975.

27. COIT. $15 / \mathrm{NC}^{\circ} 2 /$ Rév.10. Norme commerciale applicable à I'huile d'olive et à I'huile de grignons d'olive, 1-15. 2001.

28. LINCZOWSKI-BONJEAN Y. Biochimie et biologie de I'huile d'olive. Synthèse bibliographique effectuée par la societe scientifique d'hygiène alimentaire. Aliment Vie 1983 ; 2 : 47-54.

29. PAGLIARINI E, ZANONI B, GIOVANELLI G. Predictive study on tuscan virgin olive oil stability under several commercial conditions. J Agric Food Chem $2000 ; 48: 1345-51$.

30. DEBAL A. Chimie et physico-chimie des tocophérols. In : Léger $\mathrm{CL}$, ed. Vitamine E, Tocophérols et composés apparentés- Propriétés antioxygène et rôle biologique - Sources alimentaires. Paris : Polytechnica, 1992 : 1-21 ; (Coord.).

31. HARWOOD J, APARICIO R. In : Hanbook of olive oil - Analysis and properties. Maryland : Aspen Publissher, 2000 : 459-590.

32. SARTRY YSR, RAO PV, LAKSHMINARA-YANA G. Bleaching behaviour of chlorophyll substrates under vacuum on exposure to visible light. Oléagineux $1973 ; 28: 467-70$. 